\title{
Predicting mean time between failures of a maintained equipment using artificial neural network
}

\author{
V.O. Oladokun, and M.O. Shodimu \\ Department of Industrial and Production Engineering University of Ibadan, Ibadan, Nigeria \\ vo.oladokun@mail.ui.edu.ng; +2348033919050
}

\begin{abstract}
In this study an attempt is made to use the Artificial Neural Network (ANN) model predict the Mean Time between Failures of manufacturing equipment. The equipment failure pattern was carefully studied and some key factors affecting the Mean Time between Failures were identified. An Artificial Neural Network model, the Multi-Layer perceptron, with two hidden layers and seven processing elements was built. Twelve months of maintenance data of the machine was collected. The data items were divided into 3 sets: Training, Validation and Testing sets for analysis. Using the method of Back-Propagation, the Artificial Neural Network model was trained and tested. Eight input factors were identified; the output was classified into three: Low, Medium and High Mean Time Between Failures. The analysis of the model confusion matrix indicates an overall model performance accuracy of $82 \%$ with a Normalised Mean Square Error of 0.24 . It can be concluded that with the availability of adequate records, the Artificial Neural Network can serve as a useful maintenance management tool.
\end{abstract}

Keywords: Artificial Neural Network (ANN), Mean Time between Failures Multi-Layer Peceptrons (MLPs)

\section{INTRODUCTION}

The effectiveness of the maintenance function depends on the adequate planning of maintenance activities. The scheduling of maintenance activities determines the overall maintenance and operational costs of a production system. Proper planning, however, depends on the accurate estimation of relevant maintenance system parameters like Mean Time Between Failures (MTBF), Mean Time To Failures (MTTF), Mean Time To Repair, (MTTR), Reliability etc. Unfortunately, these parameters are usually influenced by multiple factors with very complex interactions and hence they are difficult to predict with the conventional mathematical or operational research models. (Oladokun e tal, 2006).

Mathematical and operation research approaches that have been used in several maintenance related problems includes such tools as Linear Programming method, Markov Model (Bobos and Protonotoriaus, 1978), Fault Tree (FT), Reliability Block Diagram (RBD) and Failure Mode and Effect Analysis (FMEA) (Telsang, 2001). These applications, however, often requires some simplifying assumptions which mostly limits their practical applictions.

In recent times the increasing availabilty and power of computing software and hardware, has encouraged the use of concepts in the areas of artificial intelligence, simulation and other computer based approaches to the maintenance problem. In this study an attempt is made at predicting the (MBTF) using the Artificial Neural Network (ANN) model. This work involves the study of the failure pattern of a major production equipment of tyre manufacturing company for the purpose of developping an ANN model for predicting the Mean Time between Failures of the equipment.

The Artificial Neural Network (ANN) is an analysis tool that is modelled after the massively parallel structure of the brain, a highly interconnected, parallel computational structure with many relatively simple individual processing elements or neurons that recieve input(s) to produce output(s). (Fadare and Fatona, 2008; Adefowoju and Osofisan, 2004; Hertz 1991)

\section{METHODOLOGY}

The company of the case study is an automobile tyre manufacturing company in Lagos, Nigeria. Data were gathered from the records of the maintenance and production departments on the accruable failures, conditions that necessitated failures, expertise of the maintenance groups responsible for repair activitities, experience of the operators, capacity of the 
equipment and other information on the working conditions relevant to the study. The methodology involves the careful observation of the various factors responsible for failures and the associated Mean Time Between Failures (MTBF). These factors were grouped as the input variables. The MTBF Failures is the output variable. Data coding to transform the data into a form suitable for Artificial Neural Network Modelling was then done.

Data Set Design and Modelling: The data set consists of input variables and an output variable. The input variables/factors are operational and

Table 1: Input Variables and the Domain

\begin{tabular}{|c|c|c|c|c|}
\hline Sn & Factor & & Domain Classification & code \\
\hline \multirow[t]{4}{*}{1} & \multirow[t]{4}{*}{ Stopage Type } & $\mathrm{F} 1$ & Mechanical Failure. & 1 \\
\hline & & $\mathrm{F} 2$ & Electrical Failure. & 2 \\
\hline & & F3 & Instrumentation. & 3 \\
\hline & & $\mathrm{F} 4$ & Services. & 4 \\
\hline \multirow[t]{3}{*}{2} & \multirow[t]{3}{*}{ Duration/ Type of Repair T } & $\mathrm{T} 1$ & $\mathrm{~T} \leq 2 \mathrm{hrs}$ & 1 \\
\hline & & T2 & $2 \mathrm{hrs}<\mathrm{T} \leq 4 \mathrm{hrs}$ & 2 \\
\hline & & T3 & $\mathrm{T}>4 \mathrm{hrs}$ & 3 \\
\hline \multirow[t]{3}{*}{3} & \multirow[t]{3}{*}{ Power supply source } & P1 & Company Generator & 1 \\
\hline & & $\mathrm{P} 2$ & Public power supply & 2 \\
\hline & & P3 & Both & 3 \\
\hline \multirow[t]{3}{*}{4} & \multirow[t]{3}{*}{ Maintenance crew of last Maintenance } & G1 & Group 1 & 1 \\
\hline & & G2 & Group2 & 2 \\
\hline & & G3 & Group3 & 3 \\
\hline \multirow[t]{2}{*}{5} & \multirow{2}{*}{$\begin{array}{l}\text { Type Spare Part used for } \\
\text { Last maintenance }\end{array}$} & S1 & Repaired/Refurbished & 1 \\
\hline & & $\mathrm{S} 2$ & New & 2 \\
\hline \multirow[t]{3}{*}{6} & \multirow[t]{3}{*}{ Production shift type } & D1 & Night Shift & 1 \\
\hline & & $\mathrm{D} 2$ & Morning Shift & 2 \\
\hline & & D3 & Afternoon & 3 \\
\hline \multirow[t]{3}{*}{7} & \multirow[t]{3}{*}{ Senior Operator in Charge } & 01 & Operator1 & 1 \\
\hline & & $\mathrm{O} 2$ & Operator 2 & 2 \\
\hline & & $\mathrm{O} 2$ & Operator 3 & 3 \\
\hline \multirow[t]{2}{*}{8} & \multirow[t]{2}{*}{ Ambient Temperature } & C1 & High above $26^{\circ} \mathrm{C}$ & 1 \\
\hline & & $\mathrm{C} 2$ & Low below $26^{\circ} \mathrm{C}$ & 2 \\
\hline \multirow[t]{2}{*}{9} & \multirow[t]{2}{*}{ Production Output } & C1 & Above Recomended capacity & 1 \\
\hline & & $\mathrm{C} 2$ & Below Recomended capacity & 2 \\
\hline
\end{tabular}

The output variable is the length of time of production before the next machine breakdown which is equivalent to MTBF. A classification approach was used as shown in Table2. The output variable for the equipment is the level of the mean time between failures of the equipment measured in the number of days between failures. environmental parameters which could influence failure occurence and length of operation before the failure occurs. Input variables inlude such as Types of Failure before last repair (stopage type), Duration of last Repair, Spare Parts for last repair or service, Production Shift, Experience of the maintenance crew of last maintenance activities, Operators on machine, Power Supply type during operation, Equipment Capacity and Weather Condition were analysed and represented in a format suitable for ANN modelling. These factors were characterised with the coresponding domain classification as shown in Table1 for the purpose quantification. 
accuracy, some are capable of handling static variables and not continuous variables. Choosing the topology for an application therefore involves trade offs of a sort.

The multi-layer perceptron with 11 hidden layers was adopted for this study chosen. Multi-layer perceptrons are feed-forward networks typically trained with static back propagation that has the ability to handle static variables for classification application suitable for approximating large input/output map.

Table2 Output Variables and Domain

\begin{tabular}{|l|l|}
\hline Output Variables & Domain \\
\hline H.MTBF & 5 days plus \\
\hline M.MTBF & 3-4days \\
\hline L.MTBF & 1-2days \\
\hline
\end{tabular}

Where

L. $M T B F=$ Low Mean Time Between Failures.

M.MTBF=Medium Mean Time Between Failures.

H. MTBF $=$ High Mean Time Between Failures.

Network training can be by Unsupervised Training or Supervised Training process. In the supervised training the desired output is given alongside the input, or by manually grading the performance of the network to get the desired output. The network process the input and compare with the desired output, errors and then back propagated through the system causing the system to adjust the weight which control the network (Dave and George, 2003).

Unsupervised training is the process by which the network provide/group output by itself from a given input. The network decides what feature it will use to group output data from the operation on the given input data, this is also known as self-organisation.

For the purpose of this study, the supervised training was applied for the network training. The data was divided into three categories; the training set, verification set and the test/validation test. The basic adjustment of connection/weight strength takes place in the training phase of the network that makes use of $60 \%$ of the data.

The Validation set used to ascertain the degree of learning between the network input and the desired output, so as to ensure that over-training or overfitting does not occur was set at $20 \%$ of data. Overtraining occurs when a network cannot generalize outcomes when it fails to perform on the data that has not been trained with, factors that could be responsible for over-training are; size of the training set, the weight and complexity of the problem (Oladokun et al 2008). Hence 60 percent of the data were used for the training set, 10 percent for the validation set and 30 percent for the test set.

\section{RESULTS}

Network Generalisation. The learning curve (figure 1) for the training processs shows that the mean square error approaches zero with increasing number of epochs indicates high level of performance of the training. Similarly in figure 2 , for the cross validation the mean square error drops with increasing number of epoch, indicating good degree of generalisation.

Network Accuracy: Test was carried out with a set of breakdown data and the neural network model applied predicted the various degree of time between failures satisfactorily as shown below in table 3 .

Table 3 Confusion Matrix

\begin{tabular}{|c|l|l|l|}
\hline $\begin{array}{l}\text { Output/ } \\
\text { Desired }\end{array}$ & L.MTBF & M.MTBF & H.MTBF \\
\hline L.MTBF & $95 \%$ & $3 \%$ & $2 \%$ \\
\hline M.MTBF & $24 \%$ & $76 \%$ & $0 \%$ \\
\hline H.MTBF & $7 \%$ & $13 \%$ & $80 \%$ \\
\hline
\end{tabular}

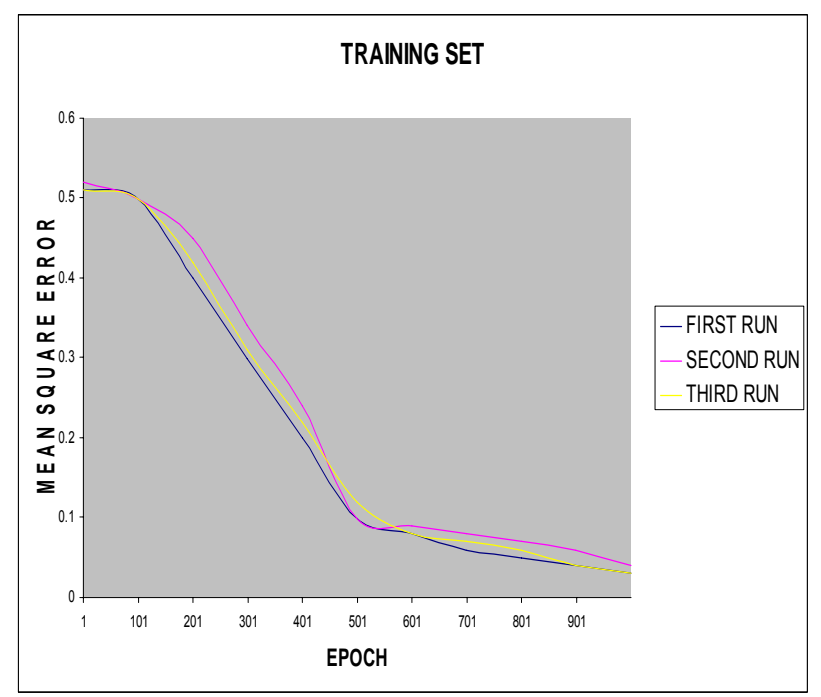

Fig 1. Training: MSE vs Epochs 
Am. J. Sci. Ind. Res., 2010, 1(3): 500-503

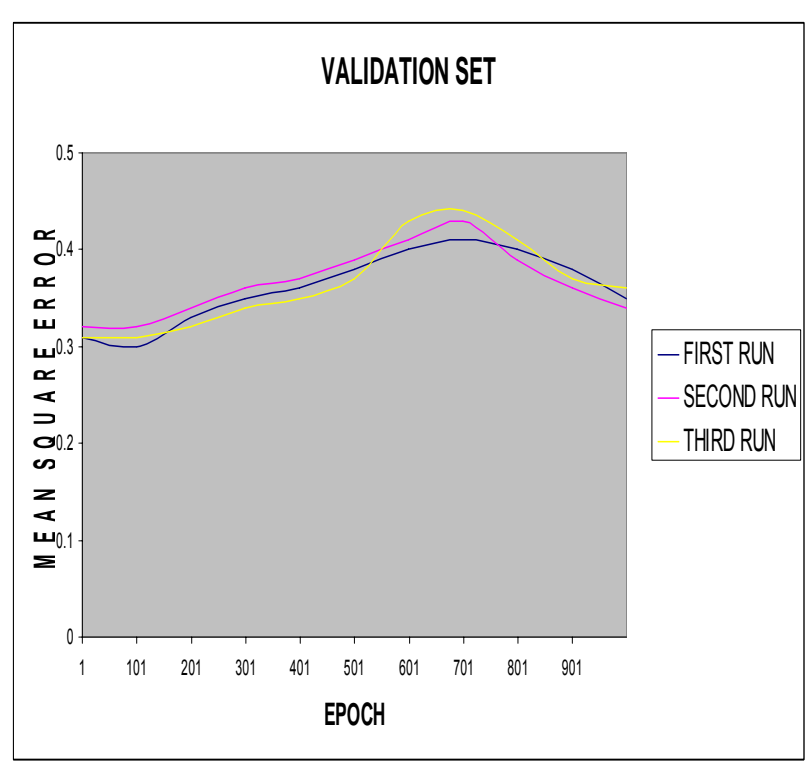

Fig 2. Validation: MSE vs Epochs

The neural network prediction of the time between failures as depicted in table 3 shows that model the accuracies of $95 \%, 76 \%$ and $80 \%$ in predicting L.MTBF, M.MTBF and H.MTBF respectively. This gives an average accuracy of over $80 \%$.

\section{CONCLUSION:}

This developed an ANN model for the prediction of the between time Between Failure of a major production equipment in an automobile tyre company the application using duration clasification approach. The model achieved an accuracy of over $80 \%$.

Hence it concluded that the application the Artificial Neural Network could be veritable tool in aiding maintenance management.

\section{REFERENCES}

1. Adefowoju, B. S. and Osofisan, A. O., 2004, Cocoa Production forecasting Using Artificial Neural Networks. International Centre for Mathematics and Computer Science Nigeria. ICMCS, 117-136.

2. Bobos, A. G. and Protonotarios, E. N, 1978., Optimal system for equipment maintenance and replacement under Markovian deterioration. European Journal of Operational Research, 2: 257-264.

3. Fadare, D.A. and A.S. Fatona. 2008. "Artificial Neural Network Modeling of Heat Transfer in a Staggered Cross-flow Tube-type Heat Exchanger". Pacific Journal of Science and Technology. 9(2):317-323.

4. Hertz, J. K., 1991. Introduction to the Theory of Neural Computation. Addison Wesley, NY.

5. Dave A. and George M. 2003. Artificial Neural Technology www. dacs.dtic. mil/techs/neural/neural_Toc.html.

6. Oladokun V.O., Charles-Owaba O.E. and Nwaozuru C.S. 2006. "An application of Artificial Neural Network to Maintenance Management". Journal of Industrial Engineering International._ 2(3): 19-26

7. Oladokun, V.O., A.T. Adebanjo, and O.E. CharlesOwaba. 2008. 'Predicting Students' Academic Performance using Artificial Neural Network: A Case Study of an Engineering Course". Pacific Journal of Science and Technology. 9(1):72-79

8. Telsang, M. 2001. "Industrial Engineering and Production Management. S. Chand \& Company Limited. 